\title{
On the semi-proper orientations of graphs
}

\author{
Ali Dehghan \\ Systems and Computer Engineering Department, Carleton University, Ottawa, Canada *
}

\begin{abstract}
A semi-proper orientation of a given graph $G$ is a function $(D, w)$ that assigns an orientation $D(e)$ and a positive integer weight $w(e)$ to each edge $e$ such that for every two adjacent vertices $v$ and $u, S_{(D, w)}(v) \neq S_{(D, w)}(u)$, where $S_{(D, w)}(v)$ is the sum of the weights of edges with head $v$ in $D$. The semi-proper orientation number of a graph $G$, denoted by $\vec{\chi}_{s}(G)$, is $\min _{(D, w) \in \Gamma} \max _{v \in V(G)} S_{(D, w)}(v)$, where $\Gamma$ is the set of all semi-proper orientations of $G$. The optimal semi-proper orientation is a semiproper orientation $(D, w)$ such that $\max _{v \in V(G)} S_{(D, w)}(v)=\vec{\chi}_{s}(G)$. In this work, we show that every graph $G$ has an optimal semi-proper orientation $(D, w)$ such that the weight of each edge is one or two. Next, we show that determining whether a given planar graph $G$ with $\vec{\chi}_{s}(G)=2$ has an optimal semi-proper orientation $(D, w)$ such that the weight of each edge is one is NP-complete. Finally, we prove that the problem of determining the semi-proper orientation number of planar bipartite graphs is NP-hard.
\end{abstract}

Key words: Proper orientation; semi-proper orientation; planar graph; optimal semi-proper orientation; bipartite graph; computational complexity.

\section{Introduction}

A directed graph $G$ is an ordered pair $(V(G), E(G))$ consisting of a set $V(G)$ of vertices and a set $E(G)$ of edges, with an incidence function $D$ that associates with each edge of $G$ an ordered pair of vertices of $G$. If $e=u v$ is an edge and $D(e)=u \rightarrow v$, then $e$ is from $u$ to $v$. The vertex $u$ is the tail of $e$, and the vertex $v$ is its head. Let $G$ be an undirected graph with no loop or parallel edges (i.e., simple graph). An orientation $D$ of a graph $G$ is a digraph obtained from the graph $G$ by replacing each edge by exactly one of the two

\footnotetext{
*E-mail address: alidehghan@sce.carleton.ca.
} 
possible arcs with the same endvertices. Also, the indegree $d_{D}^{-}(v)$ of a vertex $v$ in $D$ is the number of edges with head $v$ in $D$.

\subsection{Proper Orientation}

An orientation of a graph $G$ is called proper orientation if any two adjacent vertices have different indegrees. The proper orientation number of a graph $G$, denoted by $\vec{\chi}(G)$, is the minimum of the maximum indegree taken over all proper orientations of the graph $G$. Note that the maximum indegree of vertices is $\Delta$. On the other hand, the values of the indegrees define a proper vertex coloring of $G$ (adjacent vertices have different indegrees). Thus,

$$
\chi(G)-1 \leq \vec{\chi}(G) \leq \Delta(G)
$$

The existence of proper orientation was demonstrated by Borowiecki, Grytczuk and Pilśniak in [8], where it was shown that every graph $G$ has a proper orientation $D$ with a vertex $v$ with $d_{D}^{-}(v)=\Delta$. Afterwards, the proper orientation number was introduced in [1]. Recently, the proper orientation has been studied extensively by several authors, for instance see $[1,3,4,5,6,12]$.

We should mention that interest in proper orientations stems from their connection to the 1-2-3-Conjecture that says: "Can the edges of any non-trivial graph be assigned weights from $\{1,2,3\}$ so that adjacent vertices have different sums of incident edge weights?" [11]. For more information about 1-2-3-Conjecture and its variants see [2, 7, 9, 14].

\subsection{Semi-proper Orientation}

Motivated by the proper orientations of graphs and 1-2-3-Conjecture we investigate the semi-proper orientations of graphs. The semi-proper orientation of a given graph $G$ is a function $(D, w)$ that assigns an orientation $D(e)$ and a positive integer weight $w(e)$ to each edge $e$ such that for every two adjacent vertices $v$ and $u, S_{(D, w)}(v) \neq S_{(D, w)}(u)$, where $S_{(D, w)}(v)$ is the sum of the weights of edges with head $v$ in $D$. The semi-proper orientation number of a graph $G$, denoted by $\vec{\chi}_{s}(G)$, is $\min _{(D, w) \in \Gamma} \max _{v \in V(G)} S_{(D, w)}(v)$, where $\Gamma$ is the set of all semi-proper orientations of $G$. Note that throughout the paper for each semi-proper orientation $(D, w)$ of $G$ we denote $\sum_{z \in N_{D}^{-}(v)} w(z v)$ by $S_{(D, w)}(v)$.

Every proper orientation of a graph $G$ is a semi-proper orientation where the weights of all edges are one. Consequently, by (1), we have

$$
\chi(G)-1 \leq \vec{\chi}_{s}(G) \leq \vec{\chi}(G) \leq \Delta(G)
$$


The optimal semi-proper orientation is a semi-proper orientation $(D, w)$ such that $\max _{v \in V(G)} S_{(D, w)}(v)=\vec{\chi}_{s}(G)$. In this work, we show that every graph $G$ has an optimal semi-proper orientation $(D, w)$ such that for each edge $e \in E(G)$, we have $w(e) \in\{1,2\}$.

Theorem 1 Every graph $G$ has an optimal semi-proper orientation $(D, w)$ such that the weight of each edge is one or two.

Although for each graph $G$ we can find an optimal semi-proper orientation $(D, w)$ such that the weight of each edge is one or two, there are graphs such that they do not have any optimal semi-proper orientation without edges with label two. In other words, there are graphs such that for each of them $\vec{\chi}_{s}<\vec{\chi}$. Next, we study the complexity of finding those graphs.

Theorem 2 It is NP-complete to determine whether a given planar graph $G$ with $\vec{\chi}_{s}(G)=$ 2 has an optimal semi-proper orientation $(D, w)$ such that the weight of each edge is one.

Remark 1 Here, we show that for every tree $T$ we have $\vec{\chi}_{s}(T) \leq 2$. Let $T$ be a tree and $v$ be a vertex in $T$. Run depth-first search (DFS) algorithm from the root $v$. This defines a partition $\mathcal{L}_{0}, \mathcal{L}_{1}, \ldots, \mathcal{L}_{h}$ of the vertices of $T$ where each part $\mathcal{L}_{i}$ contains the vertices of $T$ which are at depth $i$ (i.e. at distance exactly $i$ from the vertex $v$ ). Note that by this partition each edge is between the vertices of to consecutive parts $\mathcal{L}_{j}$ and $\mathcal{L}_{j+1}$. Next, for each edge $e=u u^{\prime}$, where $u \in \mathcal{L}_{j}$ and $u^{\prime} \in \mathcal{L}_{j+1}$, orient $e$ from $u$ to $u^{\prime}$. Call the resultant orientation $D$. In orientation $D$ the indegree of each vertex except the vertex $v$ is exactly one. (Also the indegree of $v$ is zero.) Finally, define the following weight function for the edges of $T$ :

$$
w(e)= \begin{cases}1, & e=u u^{\prime}, u \in \mathcal{L}_{2 j}, u^{\prime} \in \mathcal{L}_{2 j+1}, j \geq 0 \\ 2, & \text { otherwise }\end{cases}
$$

It is easy to see that for each vertex $u \in \mathcal{L}_{2 j}$, where $j \geq 1$, we have $S_{(D, w)}(u)=2$ and for each vertex $u \in \mathcal{L}_{2 i+1}$, where $i \geq 0$, we have $S_{(D, w)}(u)=1$ and also $S_{(D, w)}(v)=0$. Thus, $(D, w)$ is a semi-proper orientation of $T$ and we have $\vec{\chi}_{s}(T) \leq 2$. This completes the proof.

It was shown [4] that it is NP-complete to decide whether the proper orientation number of a given planar bipartite graph is less than or equal to three. We improve this hardness result for semi-proper orientation number and proper orientation number of graphs. 
Theorem 3 (1) For a given planar bipartite graph $G$ determining whether $\vec{\chi}_{s}(G) \leq 2$ is NP-complete.

(2) For a given planar bipartite graph $G$ determining whether $\vec{\chi}(G) \leq 2$ is NP-complete.

The organization of the rest of the paper is as follows: In Section 2, we present some definitions and notations. Next, in Section 3, we prove that every graph $G$ has an optimal semi-proper orientation $(D, w)$ such that the weight of each edge is one or two. In Section 4, we study the computational complexity of finding optimal semi-proper orientations and computing the semi-proper orientation number of planar bipartite graphs. The paper is concluded with some remarks and open problems in Section 5

\section{Notation}

Throughout this paper we only consider finite simple graphs (i.e. finite graphs with no loop or parallel edges). We denote the vertex set and the edge set of $G$ by $V(G)$ and $E(G)$, respectively. Also, we denote the maximum degree and the minimum degree of $G$ by $\Delta(G)$ and $\delta(G)$, respectively.

A proper vertex coloring of a graph $G$ is a function $f: V(G) \rightarrow L$ such that if $v, u \in V(G)$ are adjacent, then $f(u)$ and $f(v)$ are different. A proper vertex $k$-coloring is a proper vertex coloring with $|L|=k$. The smallest integer $k$ such that the graph $G$ has a proper vertex $k$-coloring is called the chromatic number of $G$ and denoted by $\chi(G)$.

Consider the graph $G=(V, E)$, and let $U \subset V$ be any subset of vertices of $G$. Then, the induced subgraph on the set of vertices $U$ is the graph whose vertex set is $U$ and whose edge set consists of all the edges in $E$ that have both endpoints in $U$. We follow [15] for terminology and notation where they are not defined here.

\section{1-2-result for semi-proper orientations}

In this section we prove that every graph $G$ has an optimal semi-proper orientation $(D, w)$ such that the weight of each edge is one or two.

Proof of Theorem 1. We prove the theorem by using contradiction. To the contrary suppose that $G$ is a graph such that each of its optimal semi-proper orientations has an edge with weight more than two. In an optimal semi-proper orientation of a graph $G$ if the weight of an edge $e$ is more than two then we say that $e$ has a bad label in that 
semi-proper orientation. Without loss of generality suppose that each optimal semi-proper orientations of $G$ has at least $t, t>0$, edges with bad label and there is an optimal semiproper orientation such that it has exactly $t$ edges with bad label. Among all optimal semi-proper orientations of $G$, let $\mathcal{F}$ be the set of optimal semi-proper orientations such that each of them has exactly $t$ edges with bad label. Clearly, $\mathcal{F}$ is non-empty. Finally, among the optimal semi-proper orientations in $\mathcal{F}$, let $(D, w)$ be an optimal semi-proper orientation such that the sum of the weights of edges with bad labels is minimum. We assume that the optimal semi-proper orientation $(D, w)$ of $G$ has $t$ edges with bad label and the sum of the weights of edges with bad label is $b$.

Let $e=v u$ be an edge with bad label $\alpha$ in $G$ (i.e. $w(u v)=\alpha$ ) and $D(e)=u \rightarrow v$. Also, let $\mathcal{R}_{v}$ be the set of vertices such that for each of them there is directed path from the vertex $v$ to that vertex in $D$. Among all vertices in $\mathcal{R}_{v}$ let $p$ be a vertex such that the sum of weights of incoming edges to $v$ is minimum (i.e. $S_{(D, w)}(p)$ is minimum over all $\left.\left\{S_{(D, w)}(z) \mid z \in \mathcal{R}_{v}\right\}\right)$. Two cases for the vertex $p$ can be considered.

Case 1. Assume that $S_{(D, w)}(p)=S_{(D, w)}(v)$. In this case the sum of weights of incoming edges to $v$ is minimum over all vertices in $\mathcal{R}_{v}$. Let $u_{1}, \ldots, u_{k}$ be the set of vertices such that from each of them there is a directed edge to $v$. Without loss of generality assume that $u=u_{1}$. Also, let $o_{1}, \ldots, o_{r}$ be the set of vertices such that for each of them there is a directed edge from $v$ to that vertex. So, we have $o_{1}, \ldots, o_{r} \in \mathcal{R}_{v}$. Now, we study the properties of the vertex $v$.

Property 1. For each $o_{i} \in\left\{o_{1}, \ldots, o_{r}\right\}$, we have $S_{(D, w)}(v)<S_{(D, w)}\left(o_{i}\right)$.

Proof of Property 1. By our assumption for each $o_{i} \in\left\{o_{1}, \ldots, o_{r}\right\}$, we have $S_{(D, w)}(v) \leq$ $S_{(D, w)}\left(o_{i}\right)$. Since $S_{(D, w)}$ is a proper vertex coloring, for each $o_{i} \in\left\{o_{1}, \ldots, o_{r}\right\}$, we have $S_{(D, w)}(v)<S_{(D, w)}\left(o_{i}\right)$.

Property 2. For each edge $u_{i} v, 2 \leq i \leq k$, we have $w\left(u_{i} v\right)=2$.

Proof of Property 2. To the contrary assume that there is a vertex $u_{l}, 2 \leq l \leq k$, such that $w\left(u_{l} v\right) \neq 2$. Two situations can be considered.

- If $w\left(u_{l} v\right)=1$, then consider the function $\left(D^{\prime}, w^{\prime}\right)$, where

$$
w^{\prime}\left(e^{\prime}\right)= \begin{cases}w(u v)-1, & e^{\prime}=u v \\ w\left(u_{l} v\right)+1, & e^{\prime}=u_{l} v \\ w\left(e^{\prime}\right), & \text { otherwise }\end{cases}
$$


and $D^{\prime}=D$. Clearly, $\left(D^{\prime}, w^{\prime}\right)$ is an optimal semi-proper orientation such that it has at most $t$ edges with bad label and the sum of the weights of edges with bad label is less than $b$. But this is a contradiction.

- If $w\left(u_{l} v\right)>2$, then consider the function $\left(D^{\prime}, w^{\prime}\right)$, where

$$
w^{\prime}\left(e^{\prime}\right)= \begin{cases}1, & e^{\prime}=u v \\ w\left(u_{l} v\right)+w(u v)-1, & e^{\prime}=u_{l} v \\ w\left(e^{\prime}\right), & \text { otherwise }\end{cases}
$$

and $D^{\prime}=D$. Clearly, $\left(D^{\prime}, w^{\prime}\right)$ is an optimal semi-proper orientation such that it has $t-1$ edges with bad label. But this is a contradiction. This completes the proof of the Property 2.

Property 3. For each $o_{i} \in\left\{o_{1}, \ldots, o_{r}\right\}$, we have $S_{(D, w)}\left(o_{i}\right)>2 k+1$.

Proof of Property 3. We have $w\left(u_{1} v\right)=\alpha>2$, so by Property 2 , we have $S_{(D, w)}(v)>$ $2 k$. Thus, by Property 1 , we have $S_{(D, w)}\left(o_{i}\right)>2 k+1$.

Now, we are ready to prove this case. For each edge $u_{i} v, 1 \leq i \leq k$, define a variable $\operatorname{Var}\left(u_{i} v\right)$. Each variable can be one or two. The sum of variables is an integer between $k$ and $2 k$. (i.e. $k \leq \sum_{i} \operatorname{Var}\left(u_{i} v\right) \leq 2 k$ ). So the sum of the variables can be $k+1$ different integers. Consequently, there is an integer $q$ such that $k \leq q \leq 2 k$ and $q \notin\left\{S_{(D, w)}\left(u_{i}\right)\right.$ : $1 \leq i \leq k\}$. Assign one and two to the variables such that their sum is $q$. Now, define the function $\left(D^{\prime}, w^{\prime}\right)$, where

$$
w^{\prime}\left(e^{\prime}\right)= \begin{cases}\operatorname{Var}\left(u_{i} v\right), & e^{\prime}=u_{i} v, 1 \leq i \leq k, \\ w\left(e^{\prime}\right), & \text { otherwise }\end{cases}
$$

and $D^{\prime}=D$. By Property 3 , and the way that we choose $q$, it is clear that $\left(D^{\prime}, w^{\prime}\right)$ is an optimal semi-proper orientation such that it has $t-1$ edges with bad label. But this is a contradiction.

Case 2. Assume that $S_{(D, w)}(p) \neq S_{(D, w)}(v)$. By the definition of $\mathcal{R}_{v}$ there is a directed path $\mathcal{P}=v, z_{1}, \ldots, z_{l}, p$ from the vertex $v$ to the vertex $p$. Let $u_{1}, \ldots, u_{k}$ be the set of vertices such that from each of them there is a directed edge to $p$. Without loss of generality assume that $z_{l}=u_{1}$. (Note that we can have the case where the only directed path form $v$ to $p$ is the edge $\overrightarrow{v p}$. In that case we assume that $u_{1}=v$.) Next, let $o_{1}, \ldots, o_{r}$ be the set of vertices such that for each of them there is a directed edge from $p$ to that vertex. Now, 
we study the properties of the vertex $p$.

Property 4. We have $S_{(D, w)}(p) \geq w\left(u_{1} p\right)+2 k-3$.

Proof of Property 4. To the contrary suppose that $S_{(D, w)}(p) \leq w\left(u_{1} p\right)+2 k-4$. So there are indexes $j, j^{\prime}$, where $j, j^{\prime} \neq 1$ and $w\left(u_{j} p\right)=w\left(u_{j^{\prime}} p\right)=1$. First, we define a new notation and then we complete the proof. Let $z_{1}, z_{2}$ be two arbitrary adjacent vertices (i.e. $e^{\prime}=z_{1} z_{2} \in E(G)$ ). If we have $D\left(e^{\prime}\right)=z_{1} \rightarrow z_{2}$, then we denote $z_{2} \rightarrow z_{1}$ by $\neg D\left(e^{\prime}\right)$. Now, define the function $\left(D^{\prime}, w^{\prime}\right)$, where

$$
D^{\prime}\left(e^{\prime}\right)= \begin{cases}\neg D\left(e^{\prime}\right), & e^{\prime} \in \mathcal{P}, \\ D\left(e^{\prime}\right), & \text { otherwise }\end{cases}
$$

and

$$
w^{\prime}\left(e^{\prime}\right)= \begin{cases}\alpha-1, & e^{\prime}=u v \\ 1, & e^{\prime}=z_{1} v \\ w\left(v z_{1}\right), & e^{\prime}=z_{2} z_{1} \\ w\left(z_{i-1} z_{i}\right), & e^{\prime}=z_{i+1} z_{i}, 2 \leq i<l \\ w\left(z_{l-1} z_{l}\right), & e^{\prime}=p z_{l}, \\ 2, & e^{\prime}=u_{j} p \\ w\left(u_{1} p\right), & e^{\prime}=u_{j^{\prime}} p \\ w\left(e^{\prime}\right), & \text { otherwise. }\end{cases}
$$

Note that for every vertex $f$ we have $S_{(D, w)}(f)=S_{\left(D^{\prime}, w^{\prime}\right)}(f)$. Thus, $\left(D^{\prime}, w^{\prime}\right)$ is an optimal semi-proper orientation such that it has at most $t$ edges with bad label and the sum of the weights of edges with bad label is $b-1$. But this is a contradiction.

Property 5. For each $o_{i} \in\left\{o_{1}, \ldots, o_{r}\right\}$, we have $S_{(D, w)}\left(o_{i}\right)>2 k-2$. Also, we have $S_{(D, w)}\left(z_{l}\right)>2 k-2$.

Proof of Property 5. By our assumption the sum of weights of incoming edges to the vertex $p$ is minimum. Thus, for each $o_{i} \in\left\{o_{1}, \ldots, o_{r}\right\}$, we have $S_{(D, w)}(p) \leq S_{(D, w)}\left(o_{i}\right)$. Also, $S_{(D, w)}(p) \leq S_{(D, w)}\left(z_{l}\right)$. On the other hand, the function $S_{(D, w)}$ is a proper vertex coloring, so by Property 4, for each $o_{i} \in\left\{o_{1}, \ldots, o_{r}\right\}$, we have $2 k-2<S_{(D, w)}\left(o_{i}\right)$. Also, we have $2 k-2<S_{(D, w)}\left(z_{l}\right)$. 
Now, we are ready to prove Case 2 . For each edge $u_{i} p, 2 \leq i \leq k$, define a variable $\operatorname{Var}\left(u_{i} p\right)$. Each variable can be one or two. The sum of variables is an integer between $k-1$ and $2 k-2$. (i.e. $k-1 \leq \sum_{i=2}^{k} \operatorname{Var}\left(u_{i} p\right) \leq 2 k-2$ ). So the sum of the variables can be $k$ different integers. Hence, there is an integer $q$ such that $k-1 \leq q \leq 2 k-2$ and $q \notin\left\{S_{(D, w)}\left(u_{i}\right): 2 \leq i \leq k\right\}$. Assign one and two to the variables such that their sum is $q$. Now, define the function $\left(D^{\prime}, w^{\prime}\right)$, where

$$
D^{\prime}\left(e^{\prime}\right)= \begin{cases}\neg D\left(e^{\prime}\right), & e^{\prime} \in \mathcal{P}, \\ D\left(e^{\prime}\right), & \text { otherwise }\end{cases}
$$

and

$$
w^{\prime}\left(e^{\prime}\right)= \begin{cases}\alpha-1, & e^{\prime}=u v \\ 1, & e^{\prime}=z_{1} v \\ w\left(v z_{1}\right), & e^{\prime}=z_{2} z_{1} \\ w\left(z_{i-1} z_{i}\right), & e^{\prime}=z_{i+1} z_{i}, 2 \leq i<l \\ w\left(z_{l-1} z_{l}\right), & e^{\prime}=p_{l}, \\ \operatorname{Var}\left(u_{i} v\right), & e^{\prime}=u_{i} v, 2 \leq i \leq k, \\ w\left(e^{\prime}\right), & \text { otherwise. }\end{cases}
$$

By Property 5, and the way that we choose $q$, it is clear that $\left(D^{\prime}, w^{\prime}\right)$ is an optimal semi-proper orientation such that it has at most $t$ edges with bad label and the sum of the weights of edges with bad label is at most $b-1$. But this is a contradiction. This completes the proof.

\section{Hardness results}

First, we introduce planar 3-SAT (type 2) formula. Let $\Phi$ be a 3-SAT formula with the set of clauses $C=\left\{c_{1}, \cdots, c_{k}\right\}$ and the set of variables $X=\left\{x_{1}, \cdots, x_{n}\right\}$. Let $\mathcal{G}_{\Phi}$ be a graph with the set of vertices $C \cup X \cup(\neg X)$, where $\neg X=\left\{\neg x_{1}, \cdots, \neg x_{n}\right\}$, such that for each clause $c_{j}=(y \vee z \vee w)$, the vertex $c_{j}$ is adjacent to the vertices $y, z$ and $w$. Also every vertex $x_{i} \in X$ is adjacent to the vertex $\neg x_{i}$. The formula $\Phi$ is called planar 3-SAT (type 2) if the graph $\mathcal{G}_{\Phi}$ is a planar graph. Throughout the paper we refer to $\mathcal{G}_{\Phi}$ as the type 2 graph that was derived from the formula $\Phi$. It was proved that the problem of determining the satisfiability of planar 3-SAT (type 2) is NP-complete [10]. 
Problem: Satisfiability of planar 3-SAT (type 2).

InPUT: A planar 3-SAT (type 2) formula $\Phi$.

QUESTION: Is there a truth assignment for $\Phi$ that satisfies all the clauses?

Next, by using a polynomial time reduction from Satisfiability of planar 3-SAT (type 2 ), we show that it is NP-complete to determine whether a given planar graph $G$ with $\vec{\chi}_{s}(G)=2$ has an optimal semi-proper orientation $(D, w)$ such that the weight of each edge is one.

Proof of Theorem 2. Let $\Phi$ be an instance of planar 3-SAT(type 2) formula with the set of variables $X=\left\{x_{1}, \cdots, x_{n}\right\}$ and the set of clauses $C=\left\{c_{1}, \cdots, c_{k}\right\}$. We transform this formula into a planar graph $\mathcal{H}_{\Phi}$ such that $\vec{\chi}_{s}\left(\mathcal{H}_{\Phi}\right)=2$ and the graph $\mathcal{H}_{\Phi}$ has an optimal semi-proper orientation $(D, w)$ such that the weight of each edge is one if and only if there is a satisfying assignment for $\Phi$. First, we introduce two useful gadgets.

Property 6. Consider the gadget $\mathcal{T}_{x_{i}}$ which is shown in Fig. 1 . Let $(D, w)$ be an optimal semi-proper orientation of $\mathcal{T}_{x_{i}}$ such that for every edge $e$ we have $w(e)=1$. Then $\left\{S_{(D, w)}\left(x_{i}\right), S_{(D, w)}\left(\neg x_{i}\right)\right\}=\{1,2\}$.

Proof of Property 6. It is easy to see that $\vec{\chi}_{s}\left(\mathcal{T}_{x_{i}}\right)=2$. Let $(D, w)$ be an optimal semi-proper orientation of $\mathcal{T}_{x_{i}}$ such that for every edge $e$ we have $w(e)=1$. The induced subgraph on the set of three vertices $\left\{p_{2}, x_{i}, \neg x_{i}\right\}$ forms a cycle of length three. So,

$$
\left\{S_{(D, w)}\left(p_{2}\right), S_{(D, w)}\left(x_{i}\right), S_{(D, w)}\left(\neg x_{i}\right)\right\}=\{0,1,2\} .
$$

Thus, the two edges $p_{2} p_{1}$ and $p_{2} p_{3}$ were oriented from $p_{2}$ to $p_{1}$ and $p_{3}$, respectively. (Note that for every edge $e$ we have $w(e)=1$.) Therefore, $S_{(D, w)}\left(p_{1}\right)=1$ and $S_{(D, w)}\left(p_{3}\right) \in\{1,2\}$. Next, we show that $S_{(D, w)}\left(p_{3}\right)=2$. To the contrary suppose that $S_{(D, w)}\left(p_{3}\right)=1$. So, the edges $p_{3} p_{4}$ and $p_{3} p_{5}$ were oriented from $p_{3}$ to $p_{4}$ and $p_{5}$, respectively. Thus, $S_{(D, w)}\left(p_{4}\right)=$ $S_{(D, w)}\left(p_{5}\right)=1$. So for two adjacent vertices $p_{3}$ and $p_{4}$, we have $S_{(D, w)}\left(p_{3}\right)=S_{(D, w)}\left(p_{4}\right)$. But this is a contradiction. So $S_{(D, w)}\left(p_{3}\right)=2$. Consequently, $\left\{S_{(D, w)}\left(p_{1}\right), S_{(D, w)}\left(p_{3}\right)\right\}=$ $\{1,2\}$. Thus, $S_{(D, w)}\left(p_{2}\right)=0$. Hence $\left\{S_{(D, w)}\left(x_{i}\right), S_{(D, w)}\left(\neg x_{i}\right)\right\}=\{1,2\}$.

In our proof we also use the gadget $\mathcal{K}_{c_{j}}$ which is shown in Fig. 2. Next, we present the reduction.

Construction of $\mathcal{H}_{\Phi}$. We construct the planar graph $\mathcal{H}_{\Phi}$ from the type 2 graph $\mathcal{G}_{\Phi}$ (that was derived from the formula $\Phi$ ) in two steps.

Step 1. For each variable $x_{i} \in X$ put a copy of the gadget $\mathcal{T}_{x_{i}}$. We call these variable gadgets. Also, for every clause $c_{j} \in C$ put a copy of the gadget $\mathcal{K}_{c_{j}}$. We call these clause 


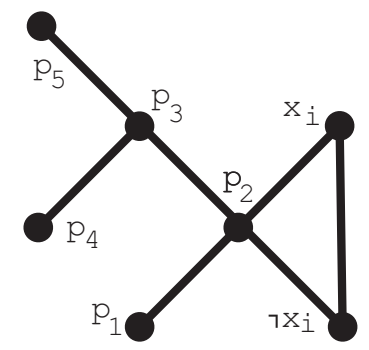

Figure 1: The gadget $\mathcal{T}_{x_{i}}$.

gadgets.

Step 2. For every clause $c_{j} \in C$ with the literals $x, y, z$ (i.e. $c_{j}=(x \vee y \vee z)$, where $x, y, z \in X \cup(\neg X))$ add the edges $x c_{1}^{j}, y c_{2}^{j}$ and $z c_{3}^{j}$. Call the resultant graph $\mathcal{H}_{\Phi}$.

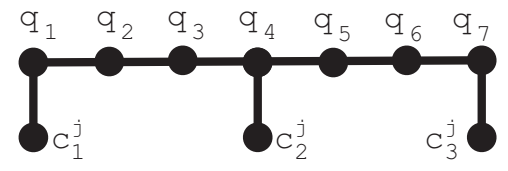

Figure 2: The gadget $\mathcal{K}_{c_{j}}$.

Clearly, the resultant graph is planar and we have $\vec{\chi}_{s}\left(\mathcal{H}_{\Phi}\right) \geq 2$. Next, we study an important property of $\mathcal{K}_{c_{j}}$.

Property 7. Let $\vec{\chi}_{s}\left(\mathcal{H}_{\Phi}\right)=2$ and $(D, w)$ be an optimal semi-proper orientation of $\mathcal{H}_{\Phi}$ such that for every edge $e$ we have $w(e)=1$. Then every edge that connects a vertex of a clause gadget to a vertex of a variable gadget is oriented from the vertex of the variable gadget to the vertex of the clause gadget.

Proof of Property 7. Let $e=x_{i} c_{1}^{j}$ be one of those edges. The induced graph on the set of three vertices $\left\{p_{2}, x_{i}, \neg x_{i}\right\}$ forms a cycle of length three. Thus,

$$
\left\{S_{(D, w)}\left(p_{2}\right), S_{(D, w)}\left(x_{i}\right), S_{(D, w)}\left(\neg x_{i}\right)\right\}=\{0,1,2\} .
$$

So, the edge $x_{i} c_{1}^{j}$ was oriented from $x_{i}$ to $c_{1}^{j}$.

Property 8. Consider the graph $\mathcal{H}_{\Phi}$. Let $c_{j} \in C$ be an arbitrary clause and $(D, w)$ be an optimal semi-proper orientation of $\mathcal{H}_{\Phi}$ such that for every edge $e$ we have $w(e)=1$. Also, assume that $\vec{\chi}_{s}\left(\mathcal{H}_{\Phi}\right)=2$. Then $2 \in\left\{S_{(D, w)}\left(c_{1}^{j}\right), S_{(D, w)}\left(c_{2}^{j}\right), S_{(D, w)}\left(c_{3}^{j}\right)\right\}$. 
Proof of Property 8. Consider the subgraph $\mathcal{K}_{c_{j}}$. To the contrary suppose that $S_{(D, w)}\left(c_{1}^{j}\right)=S_{(D, w)}\left(c_{2}^{j}\right)=S_{(D, w)}\left(c_{3}^{j}\right)=1$. By property 7 , and our assumption about the values of the function $S_{(D, w)}$ for the vertices $c_{1}^{j}, c_{2}^{j}, c_{3}^{j}$, we can conclude that the edges $c_{1}^{j} q_{1}, c_{2}^{j} q_{4}$ and $c_{3}^{j} q_{7}$ should be oriented form $c_{1}^{j},\left(c_{2}^{j}, c_{3}^{j}\right.$, respectively) to $q_{1},\left(q_{4}, q_{7}\right.$, respectively). Thus, we have $S_{(D, w)}\left(q_{1}\right)=S_{(D, w)}\left(q_{4}\right)=S_{(D, w)}\left(q_{7}\right)=2$. Since $(D, w)$ is a proper orientation we have $\left\{S_{(D, w)}\left(q_{2}\right), S_{(D, w)}\left(q_{3}\right)\right\}=\{0,1\}$ and $\left\{S_{(D, w)}\left(q_{5}\right), S_{(D, w)}\left(q_{6}\right)\right\}=\{0,1\}$. Thus the two edges $q_{3} q_{4}, q_{5} q_{4}$ were oriented from $q_{3},\left(q_{5}\right.$, respectively) to the vertex $q_{4}$. But this shows that the incoming degree of the vertex $q_{4}$ is three which is a contradiction.

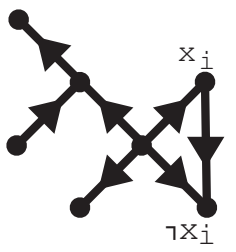

(a)

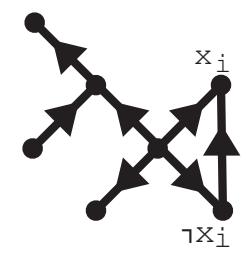

(b)

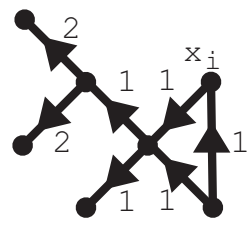

(C)

Figure 3: Three useful ways to assign orientation and weight to the edges of the gadget $\mathcal{T}_{x_{i}}$. Note that in (a) and (b) the weight of any edge is one.

Next, we show that the graph $\mathcal{H}_{\Phi}$ has an optimal semi-proper orientation $(D, w)$ such that the weight of each edge is one if and only if there is a satisfying assignment for $\Phi$.

Proof of $\Rightarrow$. Let $(D, w)$ be an optimal semi-proper orientation such that the weight of each edge is one. Let $\Gamma: X \rightarrow$ true, false be a function such that if $S_{(D, w)}\left(x_{i}\right)=1$, then $\Gamma\left(x_{i}\right)=$ true and if $S_{(D, w)}\left(x_{i}\right)=2$, then $\Gamma\left(x_{i}\right)=$ false. Next, we prove that $\Gamma$ is a satisfying assignment for $\Phi$. For each clause $c_{j}=(x \vee y \vee z)$, by Property 8 , we have $2 \in\left\{S_{(D, w)}\left(c_{1}^{j}\right), S_{(D, w)}\left(c_{2}^{j}\right), S_{(D, w)}\left(c_{3}^{j}\right)\right\}$. Thus, by Properties 6 and 7 , we have $1 \in\left\{S_{(D, w)}(x), S_{(D, w)}(y), S_{(D, w)}(z)\right\}$. Consequently, the function $\Gamma$ is a satisfying assignment for $\Phi$.

Proof of $\Leftarrow . \quad$ Assume that $\Phi$ is satisfiable with the satisfying assignment $\Gamma: X \rightarrow$ \{true, false\}. Next, in three steps, we present an optimal semi-proper orientation such that the weight of each edge is one.

- If $e$ is an edge that connects a vertex of a clause gadget to a vertex of a variable gadget, then orient $e$ from the vertex of the variable gadget to the vertex of the clause gadget.

- For each gadget $\mathcal{T}_{x_{i}}$ if $\Gamma(x)=$ true, then orient the edges of $\mathcal{T}_{x_{i}}$ like Fig. 3.(a). Otherwise 
orient those edges like Fig. 3.(b).

- For each gadget $\mathcal{K}_{c_{j}}$, where $c_{j}=(x \vee y \vee z)$, we have $1 \in\left\{S_{(D, w)}(x), S_{(D, w)}(y), S_{(D, w)}(z)\right\}$. Consequently, we have $2 \in\left\{S_{(D, w)}\left(c_{1}^{j}\right), S_{(D, w)}\left(c_{2}^{j}\right), S_{(D, w)}\left(c_{3}^{j}\right)\right\}$. Thus base on the values of $S_{(D, w)}\left(c_{1}^{j}\right), S_{(D, w)}\left(c_{2}^{j}\right), S_{(D, w)}\left(c_{3}^{j}\right)$ use one of the orientations that were presented in Fig. 4. This completes the proof of $(\Leftarrow)$.

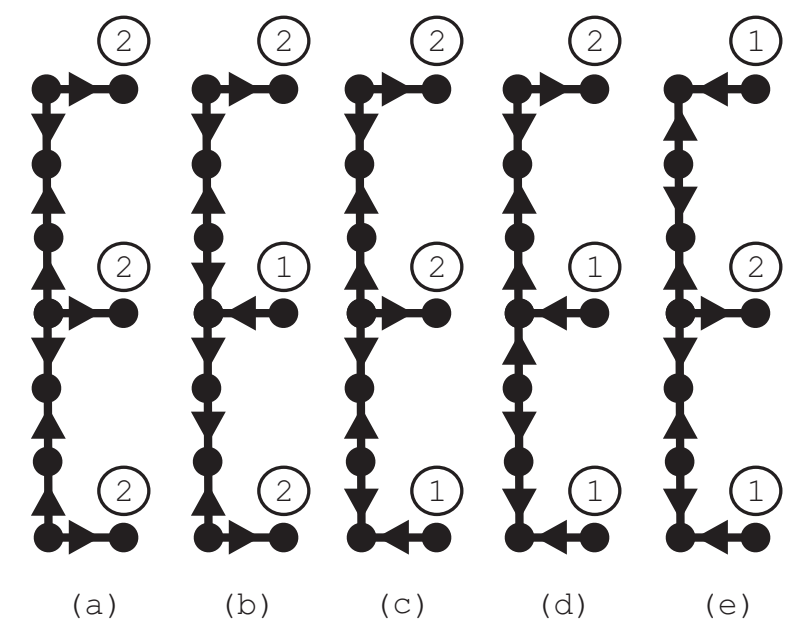

Figure 4: Possible ways to assign orientations to the edges of the gadget $\mathcal{K}_{c_{j}}$.

Finally, we show that the semi-proper orientation number of the graph $\mathcal{H}_{\Phi}$ is always two. We present an optimal semi-proper orientation $(D, w)$ such that the weights of some edges are two. Consider the following orientations and weights for the edges of the graph. - If $e$ is an edge that connects a vertex of a clause gadget to a vertex of a variable gadget, then put $w(e)=1$ and orient $e$ from the vertex of the variable gadget to the vertex of the clause gadget.

- For each gadget $\mathcal{T}_{x_{i}}$ put orientations and weights on the edges of $\mathcal{T}_{x_{i}}$ like Fig. 3.(c)

- For each gadget $\mathcal{K}_{c_{j}}$, put the weight of any edge one and orient them like Fig. 4.(a). This completes the proof.

In Part (1) of the next proof we show that for a given planar bipartite graph $G$ determining whether $\vec{\chi}_{s}(G) \leq 2$ is NP-complete. After that in Part (2), we show that computing $\vec{\chi}_{s}(G)$ for planar bipartite graphs is NP-hard.

Proof of Theorem 3. (1) It is clear that the problem is in NP. We reduce Cubic planar 1-in-3 $S A T$ to our problem in polynomial time. 
Cubic planar 1-in-3 SAT.

InstANCE: A 3-SAT formula $\Phi=(X, C)$ such that every variable appears in exactly three clauses, there is no negation in the formula, and the bipartite graph obtained by linking a variable and a clause if and only if the variable appears in the clause, is planar.

Question: Is there a truth assignment for $X$ such that each clause in $C$ has exactly one true literal?

In 2001, Moore and Robson proved that Cubic planar 1-in-3 SAT is NP-complete [13].

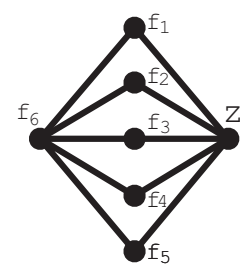

Figure 5: The gadget $\mathcal{F}_{1}$.

Consider an instance $\varphi$ of Cubic planar 1-in-3 SAT. We transform this into a planar bipartite graph $G$ such that the semi-proper orientation number of $G$ is two if and only if the formula $\varphi$ has a 1-in-3 assignment. First, we introduce some useful gadgets.

Property 9. Let $G$ be graph such that $\vec{\chi}_{s}(G)=2$ and it has the gadget $\mathcal{F}_{1}$ (which is shown in Fig. 5) as an induced subgraph. Also, let $(D, w)$ be an optimal semi-proper orientation of $G$. Then $S_{(D, w)}(z)=\{0,1\}$.

Proof of Property 9. To the contrary suppose that $S_{(D, w)}(z)=2$. So by the symmetry of the gadget without loss of generality we can assume that the edges $z f_{1}, z f_{2}, z f_{3}$ were oriented from $z$ to $f_{1}\left(f_{2}\right.$ and $f_{3}$, respectively). Since $S_{(D, w)}$ is a proper vertex coloring, we have $S_{(D, w)}\left(f_{1}\right)=S_{(D, w)}\left(f_{2}\right)=S_{(D, w)}\left(f_{3}\right)=1$. Thus, the three edges $f_{1} f_{6}, f_{2} f_{6}, f_{3} f_{6}$ were oriented form $f_{1}\left(f_{2}, f_{3}\right.$, respectively) to $f_{6}$. Hence $S_{(D, w)}\left(f_{6}\right) \geq 3$. But this is a contradiction. This completes the proof.

Property 10. Let $G$ be graph such that $\vec{\chi}_{s}(G)=2$ and it has the gadget $\mathcal{F}_{2}$ (which is shown in Fig. 6) as an induced subgraph. Also, let $(D, w)$ be an optimal semi-proper orientation of $G$. Then $S_{(D, w)}\left(z_{2}\right)=0$.

Proof of Property 10. By Property 9, we have $S_{(D, w)}\left(z_{1}\right), S_{(D, w)}\left(z_{2}\right), S_{(D, w)}\left(z_{3}\right) \in$ $\{0,1\}$. To the contrary suppose that $S_{(D, w)}\left(z_{2}\right)=1$. Then at least one of the edges 
$z_{1} z_{2}, z_{2} z_{3}$ were oriented form $z_{2}$ to the other endpoint. Without loss of generality assume that $z_{2} z_{3}$ were oriented form $z_{2}$ to $z_{3}$. So, $S_{(D, w)}\left(z_{2}\right)=S_{(D, w)}\left(z_{3}\right)=1$. But this is a contradiction.

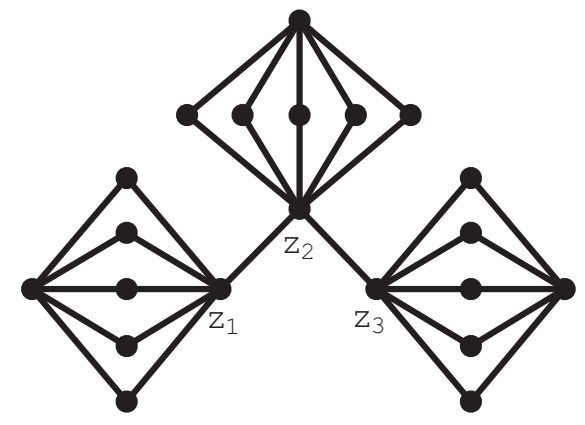

Figure 6: The gadget $\mathcal{F}_{2}$.

Property 11. Let $G$ be graph such that $\vec{\chi}_{s}(G)=2$ and it has the variable gadget $\mathcal{H}_{x_{i}}$ (which is shown in Fig. 7) as an induced subgraph. Also, let $(D, w)$ be an optimal semi-proper orientation of $G$. Then $\left\{S_{(D, w)}\left(x_{i}\right), S_{(D, w)}\left(\neg x_{i}\right)\right\}=\{1,2\}$.

Proof of Property 11. By Property 10, we have $S_{(D, w)}\left(z_{2}\right)=S_{(D, w)}\left(z_{2}^{\prime}\right)=0$. Thus, the edges $z_{2} x_{i}$ and $z_{2}^{\prime} \neg x_{i}$ were oriented from $z_{2}\left(z_{2}^{\prime}\right.$, respectively) to $x_{i}\left(\neg x_{i}\right.$, respectively). Consequently, $\left\{S_{(D, w)}\left(x_{i}\right), S_{(D, w)}\left(\neg x_{i}\right)\right\}=\{1,2\}$.

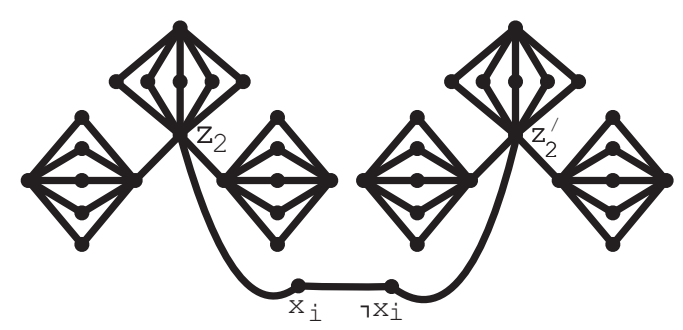

Figure 7: The gadget $\mathcal{H}_{x_{i}}$.

Property 12. Let $G$ be graph such that $\vec{\chi}_{s}(G)=2$ and it has the gadget $\mathcal{Q}_{c_{j}}$ (which is shown in Fig. 8) as an induced subgraph. Also, let $(D, w)$ be an optimal semi-proper orientation of $G$. If there are two edges $x c_{1}^{j}$ and $y c_{2}^{j}$ such that $D\left(x c_{1}^{j}\right)=x \rightarrow c_{1}^{j}$ and $D\left(y c_{2}^{j}\right)=y \rightarrow c_{2}^{j}$. Then $2 \in\left\{S_{(D, w)}\left(c_{1}^{j}\right), S_{(D, w)}\left(c_{2}^{j}\right)\right\}$.

Proof of Property 12. By Properties 9 and 10, we have $S_{(D, w)}\left(z_{1}\right)=1$. On the other 
hand, by Property 8, we should have $2 \in\left\{S_{(D, w)}\left(z_{1}\right), S_{(D, w)}\left(c_{1}^{j}\right), S_{(D, w)}\left(c_{2}^{j}\right)\right\}$. (Note that although in Property 8, we assume that the weight of any edge is one, the proof for the case where the weights are in $\{1,2\}$ is still correct.) Thus, we have $2 \in\left\{S_{(D, w)}\left(c_{1}^{j}\right), S_{(D, w)}\left(c_{2}^{j}\right)\right\}$.

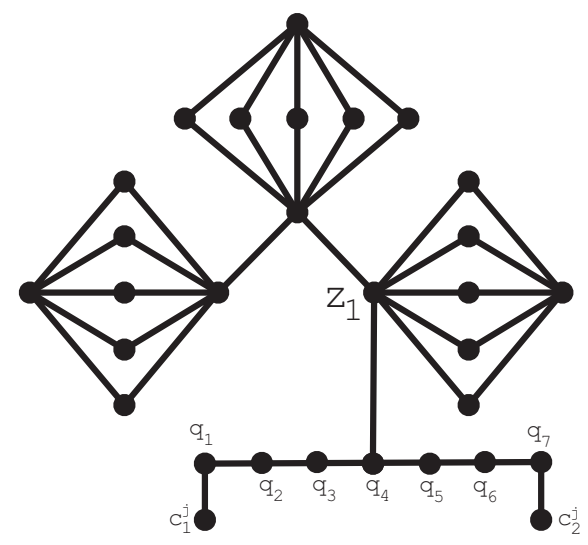

Figure 8: The gadget $\mathcal{Q}_{c_{j}}$.

Property 13. Let $G$ be graph such that $\vec{\chi}_{s}(G)=2$ and it has the gadget $\mathcal{L}_{c_{j}}$ (which is shown in Fig. 9) as an induced subgraph. Also, let $(D, w)$ be an optimal semi-proper orientation of $G$. Then $\left\{S_{(D, w)}\left(c_{1}^{j}\right), S_{(D, w)}\left(c_{2}^{j}\right), S_{(D, w)}\left(c_{3}^{j}\right)\right\} \neq\{2,2,2\}$.

Proof of Property 13. To the contrary assume that $\left\{S_{(D, w)}\left(c_{1}^{j}\right), S_{(D, w)}\left(c_{2}^{j}\right), S_{(D, w)}\left(c_{3}^{j}\right)\right\}=$ $\{2,2,2\}$. The semi-proper orientation number of $G$ is two, so at least one of the three edges $p q_{2}, p q_{4}, p q_{6}$ was oriented from $p$ to the other endpoint. By the symmetry suppose that $p q_{2}$ was oriented from $p$ to $q_{2}$. On the other hand, by property 9 , we have $S_{(D, w)}\left(q_{2}\right) \in\{0,1\}$. Thus, $S_{(D, w)}\left(q_{2}\right)=1$. So, the edge $q_{2} q_{1}$ was oriented from $q_{2}$ to $q_{1}$. Thus, $S_{(D, w)}\left(q_{1}\right)=2$, but this is a contradiction with $\left\{S_{(D, w)}\left(c_{1}^{j}\right), S_{(D, w)}\left(c_{2}^{j}\right), S_{(D, w)}\left(c_{3}^{j}\right)\right\}=\{2,2,2\}$. This completes the proof.

Property 14. Let $G$ be graph such that $\vec{\chi}_{s}(G)=2$ and it has the clause gadget $\mathcal{S}_{c_{j}}$ (which is shown in Fig. 10) as an induced subgraph. Also, let $(D, w)$ be an optimal semi-proper orientation of $G$. Then $\left\{S_{(D, w)}\left(c_{1}^{j}\right), S_{(D, w)}\left(c_{2}^{j}\right), S_{(D, w)}\left(c_{3}^{j}\right)\right\}=\{1,2,2\}$.

Proof of Property 14. By Properties 12 and 13, the proof is clear.

Now, we present the construction of $G$ and prove the reduction. Construction of $G$. We construct the planar bipartite graph $G$ from $\varphi$ in two steps.

Step 1. For each variable $x_{i} \in X$ put a copy of the variable gadget $\mathcal{H}_{x_{i}}$, which is shown 


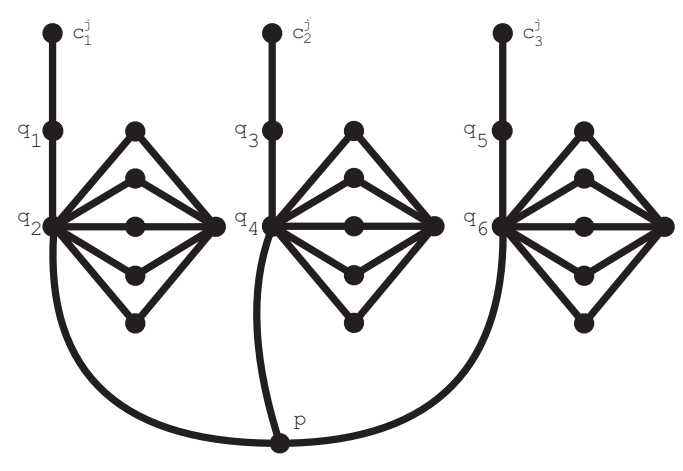

Figure 9: The gadget $\mathcal{L}_{c_{j}}$.

in Fig. 7. Also, for every clause $c_{j} \in C$ put a copy of the clause gadget $\mathcal{S}_{c_{j}}$, which is shown in Fig. 10.

Step 2. For every clause $c_{j} \in C$ with the variables $x, y, z$ add the edges $x c_{1}^{j}, y c_{2}^{j}$ and $z c_{3}^{j}$. Call the resultant graph $G$. It is easy to check that $G$ is planar and bipartite.

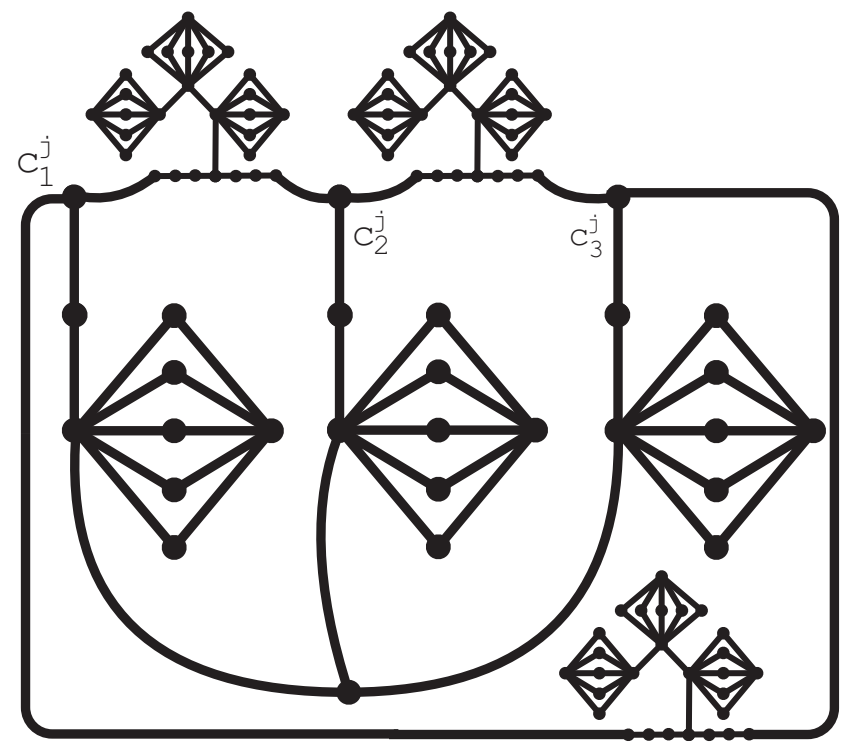

Figure 10: The clause gadget $\mathcal{S}_{c_{j}}$.

Next, we show that the semi-proper orientation number of the graph $G$ is two if and only if there is a 1-in-3 satisfying assignment for $\varphi$.

Proof of $\Rightarrow$. Let $\vec{\chi}_{s}(G)=2$ and $(D, w)$ be an optimal semi-proper orientation. Let 
$\Gamma: X \rightarrow$ true, false $\}$ be a function such that if $S_{(D, w)}\left(x_{i}\right)=2$, then $\Gamma\left(x_{i}\right)=$ true and if $S_{(D, w)}\left(x_{i}\right)=1$, then $\Gamma\left(x_{i}\right)=$ false. For each clause $c_{j}=(x \vee y \vee z)$, by Property 14, we have

$$
\left\{S_{(D, w)}\left(c_{1}^{j}\right), S_{(D, w)}\left(c_{2}^{j}\right), S_{(D, w)}\left(c_{3}^{j}\right)\right\}=\{1,2,2\}
$$

So,

$$
\left\{S_{(D, w)}(x), S_{(D, w)}(y), S_{(D, w)}(z)\right\}=\{2,1,1\} .
$$

Thus, the function $\Gamma$ is a 1-in-3 satisfying assignment for $\varphi$.

Proof of $\Leftarrow$. Assume that $\varphi$ is satisfiable with the 1-in-3 satisfying assignment $\Gamma: X \rightarrow$ \{true, false . For each variable gadget $\mathcal{H}_{x_{i}}$ orient its edges such that $S_{(D, w)}\left(x_{i}\right)=2$ if and only if $\Gamma\left(x_{i}\right)=$ true. Next, for each edge $e$ that connects a vertex of a clause gadget to a vertex of a variable gadget, put $w(e)=1$ and orient $e$ from the vertex of the variable gadget to the vertex of the clause gadget. Finally we need orient the clause gadgets. Note that for each clause gadget $\mathcal{S}_{c_{j}}$, we have $\left\{S_{(D, w)}\left(c_{1}^{j}\right), S_{(D, w)}\left(c_{2}^{j}\right), S_{(D, w)}\left(c_{3}^{j}\right)\right\}=\{1,2,2\}$. Thus, we can use the orientation which is presented in Fig. 11 and Fig. 12 to find an optimal semi-proper orientation for clause gadgets. This completes the proof of Part (1).

(2) Note that in the proof of Part (1), we never assign weight one to each edge. Thus, the proof of that part shows the NP-hardness of Part (2) and completes the proof.

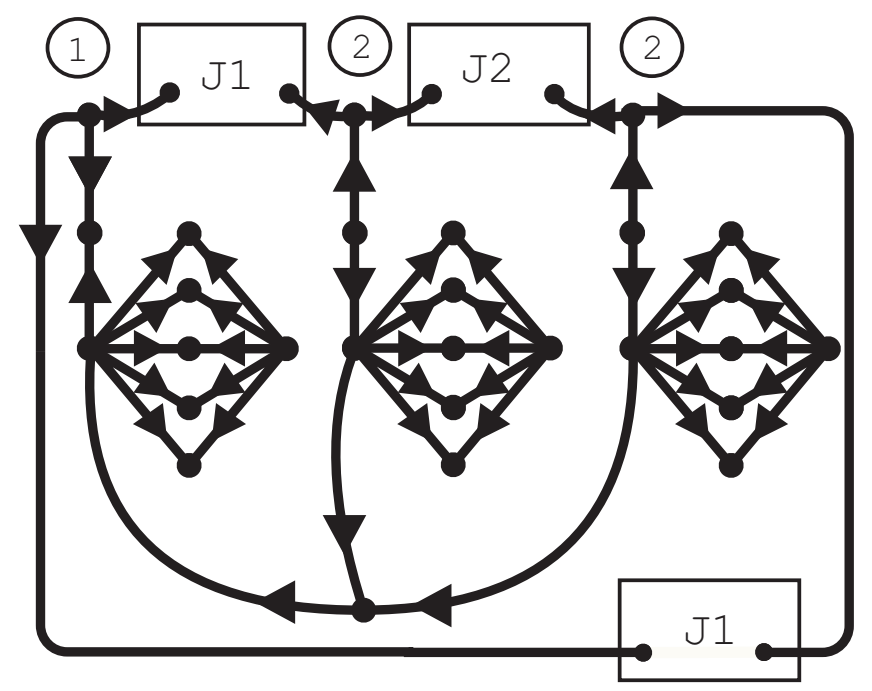

Figure 11: An optimal semi-proper orientation for the gadget $\mathcal{S}_{c_{j}}$, where the weight of each edge is one and the orientations of the subgraphs $J 1$ and $J 2$ are presented in Fig. 12. 


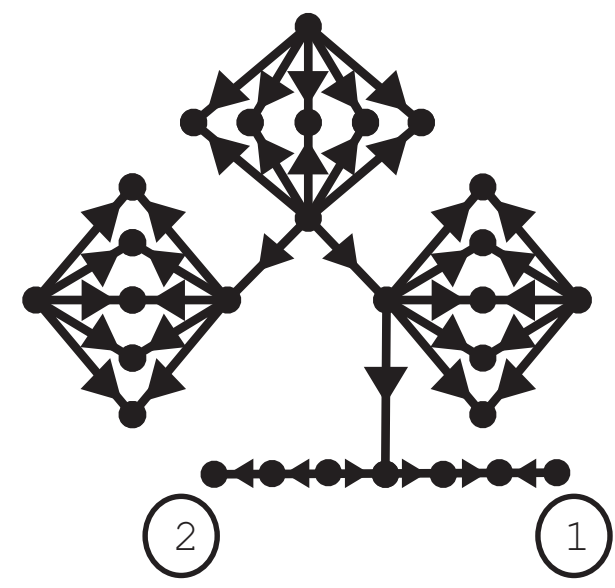

J1

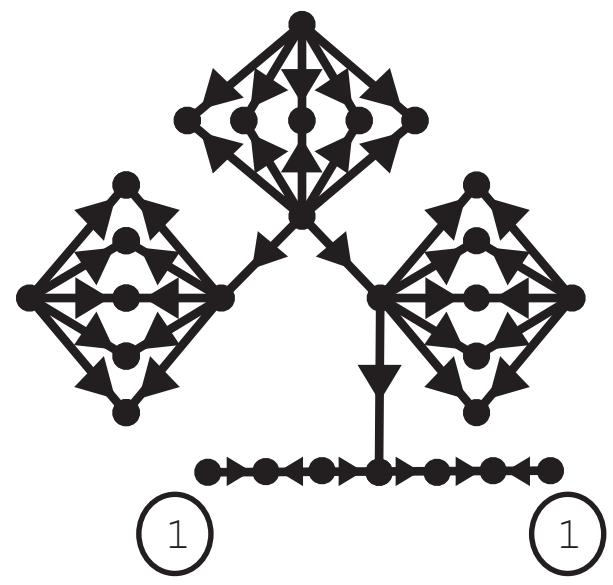

J2

Figure 12: An optimal semi-proper orientation for the gadgets $J 1$ and $J 2$, where the weight of each edge is one.

\section{Conclusions and future research}

- In this work, we introduced the notation of semi-proper orientations of graphs and studied some properties of these orientations. It is easy to see that $\vec{\chi}_{s}(G) \leq \vec{\chi}(G)$. There are several questions regarding the relationship between the proper orientation number and the semi-proper orientation number of graphs. We pose some of them here.

Problem 1. Is there any constant number $c$ such that $\vec{\chi}(G)-\vec{\chi}_{s}(G) \leq c$ ?

Problem 2. Is there any important family of graphs such that for graphs in that family there is a polynomial time algorithm to compute $\vec{\chi}_{s}(G)$ but computing $\vec{\chi}(G)$ is NP-hard?

- The problem of determining an upper bound for the proper orientation numbers of planar graphs is a well-known open problem in this area [4, 6]. We pose the same problem.

Problem 3. Does there exist a constant number $c$ such that $\vec{\chi}_{s}(G) \leq c$ for all planar graphs $G$ ?

- We proved that every graph $G$ has an optimal semi-proper orientation $(D, w)$ such that the weight of each edge is one or two. Also, we proved that determining whether a 
given planar graph $G$ with $\vec{\chi}_{s}(G)=2$ has an optimal semi-proper orientation $(D, w)$ such that the weight of each edge is one is NP-complete. It is interesting to find some families of graphs such that each graph in those families has an optimal semi-proper orientation $(D, w)$ such that the weight of each edge is one. We pose the following problem.

Problem 4. Is there any polynomial time algorithm to determine whether a given bipartite graph $G$ has an optimal semi-proper orientation $(D, w)$ such that the weight of each vertex is one?

- Furthermore, in this work we proved that the problem of determining the semi-proper orientation number of planar bipartite graphs is NP-hard. Regarding the complexity of computing the proper orientation number of regular graphs it was shown in [1] that it is NP-complete to decide whether the proper orientation number of a given 4-regular graph is 3 . What can we say about the complexity of computing the semi-proper orientation number of regular graphs?

Problem 5. Determine the computational complexity of computing the semi-proper orientation number of regular graphs.

\section{References}

[1] A. Ahadi and A. Dehghan. The complexity of the proper orientation number. Inform. Process. Lett., 113(19-21):799-803, 2013.

[2] A. Ahadi, A. Dehghan, M. Kazemi, and E. Mollaahmadi. Computation of lucky number of planar graphs is NP-hard. Inform. Process. Lett., 112(4):109-112, 2012.

[3] A. Ahadi, A. Dehghan, and M. Saghafian. Is there any polynomial upper bound for the universal labeling of graphs? J. Comb. Optim., 34(3):760-770, 2017.

[4] J. Araújo, N. Cohen, S. F. de Rezende, F. Havet, and P. F. S. Moura. On the proper orientation number of bipartite graphs. Theoret. Comput. Sci., 566:59-75, 2015.

[5] J. Araujo, F. Havet, C. Linhares Sales, and A. Silva. Proper orientation of cacti. Theoret. Comput. Sci., 639:14-25, 2016.

[6] J. Araújo, C. L. Sales, I. Sau, and A. Silva. Weighted proper orientations of trees and graphs of bounded treewidth. Theoret. Comput. Sci., accepted, 2018. 
[7] P. Bennett, A. Dudek, A. Frieze, and L. Helenius. Weak and strong versions of the 1-2-3 conjecture for uniform hypergraphs. Electron. J. Combin., 23(2):Paper 2.46, $21,2016$.

[8] M. Borowiecki, J. Grytczuk, and M. Pilśniak. Coloring chip configurations on graphs and digraphs. Inform. Process. Lett., 112(1-2):1-4, 2012.

[9] D. W. Cranston, S. Jahanbekam, and D. B. West. The 1,2,3-conjecture and 1,2conjecture for sparse graphs. Discuss. Math. Graph Theory, 34(4):769-799, 2014.

[10] D.-Z. Du, K.-K Ko, and J. Wang. Introduction to Computational Complexity. Higher Education Press, 2002.

[11] M. Karoński, T. Ł uczak, and A. Thomason. Edge weights and vertex colours. J. Combin. Theory Ser. B, 91(1):151-157, 2004.

[12] F. Knox, N. Matsumoto, S. G. Hermosillo de la Maza, B. Mohar, and C. Linhares Sales. Proper orientations of planar bipartite graphs. Graphs Combin., 33(5):1189-1194, 2017.

[13] C. Moore and J. M. Robson. Hard tiling problems with simple tiles. Discrete Comput. Geom., 26(4):573-590, 2001.

[14] B. Seamone and B. Stevens. Sequence variations of the 1-2-3 conjecture and irregularity strength. Discrete Math. Theor. Comput. Sci., 15(1):15-28, 2013.

[15] D. B. West. Introduction to graph theory. Prentice Hall Inc., Upper Saddle River, NJ, 1996. 